\title{
Discussion on Diagnostic Value of D-dimer and Troponin POCT in Emergency Department for Fatal Chest Pain Caused by Acute Myocardial Infarction and Acute Aortic Dissection
}

\author{
Xingguo Peng ${ }^{1}$, Gongchun Fan ${ }^{2,}$, Kun Zhang ${ }^{3}$ \\ ${ }^{1}$ Center of Emergency and Critical Care, Renmin Hospital, Hubei University of Medicine, \\ Shiyan Renmin Hospital, Shiyan, Hubei Province, China,442000 \\ ${ }^{2}$ Department of Respiratory Medicine, Renmin Hospital, Hubei University of Medicine, Shiyan \\ Renmin Hospital, Shiyan, Hubei Province, China,442000 \\ ${ }^{3}$ The First Clinical College of Wuhan University, Wuhan, Hubei Province, China, 430000 \\ *Corresponding author E-mail: hubeiwenbo@163.com \\ Address: Chaoyang District Road No. 39, Shiyan City, Hubei Province, P.R.China, 442000
}

Keywords: emergency department; POCT; D-dimer; troponin; acute myocardial infarction; acute aortic dissection; fatal chest pain; diagnostic value

Abstract: Objective: to summarize and analyze application value of Point Of Care Testing (POCT) of D-dimer and troponin in diagnosis and identification of fatal chest pain caused by acute myocardial infarction and acute aortic dissection. Method: 120 patients with chest pain who were received and treated by the emergency department from June 2015 to May 2018 were chosen as the objects of study. D-dimer and troponin POCT was implemented for all patients. Finally, 58 patients were definitely diagnosed with acute myocardial infarction, and 62 patients were definitely diagnosed with acute aortic dissection. The level of D-dimer and troponin was compared and analyzed for both groups. Results: D-dimer level of patients with acute aortic dissection was higher than that of patients with acute myocardial infarction. Troponin level of patients with acute aortic dissection was lower than that of patients with acute myocardial infarction. The comparison of the above index differences had statistical significance $(\mathrm{P}<0.05)$. Conclusion: rapid bedside detection of $\mathrm{D}$-dimer and troponin can effectively identify and diagnose fatal chest pain caused by acute myocardial infarction and acute aortic dissection, provides basis for early rescue and treatment and improve patients’ prognosis, so it deserves to be promoted and applied.

Acute coronary syndrome, pulmonary embolism, aortic dissection, acute myocardial infarction, cardiac tamponade and tension pneumothorax can cause fatal chest pain. The above diseases are characterized by sudden onset and fast disease changes. Timely rescue and treatment can help patients eliminate symptoms, and standard treatment can make sure patients with different diseases can receive treatment within a short time ${ }^{[1]}$. The development of POCT allows more acute disease patients to complete diagnosis at bedside and provides basis for 
treatment ${ }^{[2]}$. The identification and diagnostic value of D-dimer and troponin POCT was explored by combining 120 patients with chest pain who were received and treated in the emergency department from June 2015 to May 2018. The content analysis is as follows:

\section{Clinical data and method}

\subsection{General data}

120 patients with chest pain who were received and treated by the emergency department from June 2015 to May 2018 were chosen as the objects of study. All patients were definitely diagnosed. After coronary angiogram examination, 58 patients were definitely diagnosed with acute myocardial infarction, including 34 male patients and 24 female patients. The age of patients was 34-78, with the average age of $60.45 \pm 4.55$. 62 patients were definitely diagnosed with acute aortic dissection by aorta CTA, including 36 male patients and 26 female patients. The age of patients was 35-77, with the average age of $59.97 \pm 4.63$. The general data differences of both groups were not obvious, without statistical significance $(\mathrm{P}>0.05)$.

\subsection{Inclusion criteria and exclusion criteria}

Inclusion criteria: (1) patients finally definitely diagnosed with acute myocardial infarction or acute aortic dissection; (2) implementation of D-dimer and troponin POCT; (3) complete follow-up visit data; (4) approved by Ethics Committee. Exclusion criteria: (1) autoimmune disease; (2) patients with thyroid function abnormality, atrial fibrillation, infection and malignant tumor; (3) patients with liver, kidney, heart and lung insufficiency, mental disorder and varicosity, and those lying in bed for a long time; (4) orally took anticoagulant before hospitalization.

\subsection{Method}

POCT was implemented for all patients with fatal chest pain. The detection items included D-dimer and troponin. Fluorescence immunoanalyzer was applied for D-dimer detection, with the model of HTY-100. The detection was completed quickly as per the instructions. Enzyme-linked immunosorbent assay was adopted for troponin detection. The detection was completed in strict accordance with instructions of troponin I detection kit.

\subsection{Observation indexes}

Observation indexes were the content of D-dimer and troponin of patients. D-dimer content $>600 \mathrm{ng} / \mathrm{ml}$ means rise, and troponin content $>0.40 \mathrm{ng} / \mathrm{ml}$ means rise ${ }^{[3]}$.

\subsection{Statistical analysis}

Statistical software SPSS21.0 was used for data analysis. D-dimer and troponin content was expressed with mean \pm standard deviation. $t$ test was applied for difference comparison. Standard of statistical significance: $\mathrm{P}<0.05$.

\section{Results}

D-dimer and troponin content comparison of both groups is shown in Table 1. According to the data in Table 1, D-dimer content of patients with acute myocardial infarction was lower 
than that of patients with acute aortic dissection. Troponin content of patients with acute myocardial infarction was higher than that of patients with acute aortic dissection. The difference comparison had statistical significance $(\mathrm{P}<0.05)$. The data are shown in Table 1 .

Table $1 \mathrm{D}$-dimer and troponin content of patients with acute myocardial infarction and patients with acute aortic dissection $\left(\mathrm{X}_{ \pm \mathrm{s}}\right)$

\begin{tabular}{cccc}
\hline Group & No. & D-dimer $(\mathrm{ng} / \mathrm{ml})$ & Troponin $(\mathrm{ng} / \mathrm{ml})$ \\
\hline Acute myocardial infarction & 58 & $162.63 \pm 44.26$ & $0.60 \pm 1.18$ \\
Acute aortic dissection & 62 & $520.81 \pm 79.48$ & $0.10 \pm 0.42$ \\
$\mathrm{t}$ & $/$ & 30.212 & 3.131 \\
$\mathrm{P}$ & $/$ & $<0.05$ & $<0.05$ \\
\hline
\end{tabular}

\section{Discussion}

Clinically, multiple diseases can cause fatal chest pain, so it is necessary to clearly know the pathogeny, identify and diagnose primary foundation before treatment of fatal chest pain. Then, basic treatment can be done well according to the diagnosis result for the implementation of standard treatment. Acute myocardial infarction and acute aortic dissection are the primary causes for fatal chest pain. Effective identification and diagnosis of the above two diseases have important significance for follow-up rescue. For both patients with acute myocardial infarction and patients with acute aortic dissection, they have typical manifestations of fatal chest pain after onset in the early stage. Other relevant early manifestations lack specificity. Thus, acute myocardial infarction and acute aortic dissection cannot be identified only by depending on clinical symptoms. Many methods can be used to identify acute myocardial infarction and acute aortic dissection, such as ultrasonic cardiogram examination, blood vessel CT examination, contrast examination and electrocardiographic examination. Although there are many examination method available, in view of rapid rescue after the onset, early examination and diagnosis method should be easy to operate, and the results should come out quickly. In view of time consumption or complex operation limitations of the above diagnostic methods, POCT laboratory indexes have been gradually applied to identify and diagnose primary disease of fatal chest pain ${ }^{[4]}$.

In this study, D-dimer and troponin indexes were used to identify and diagnose fatal chest pain. In the later period, other methods were used to definitely diagnose the disease. Through comparison and analysis of laboratory parameters, D-dimer content of patients with acute myocardial infarction was obviously lower than that of patients with acute aortic dissection, promoting D-dimer level of patients with acute aortic dissection rises obviously. In terms of troponin index, troponin content of patients with acute myocardial infarction was obviously higher than that of patients with acute aortic dissection. The above two indexes can serve as the important thoughts and methods to identify and diagnose acute myocardial infarction and acute aortic dissection. D-dimer and troponin used for diagnosis of fatal chest pain have important clinical significance. The change of D-dimer content can be used as an important marker to judge hypercoagulable state and hyperfibrinolysis. Acute myocardial infarction results from severe stenosis or blockage of coronary artery branches, so ischemic necrosis happens to myocardium. Wang Zhichao et al. reported the application value of plasma D-dimer in the diagnosis of acute myocardial infarction, acute pulmonary embolism and acute aortic dissection. The data analysis showed that, D-dimer level of patients with acute myocardial infarction was obviously lower than that of patients with acute pulmonary embolism and acute aortic dissection. The results hold that, D-dimer can be used to identify acute myocardial 
infarction, acute pulmonary embolism and acute aortic dissection and avoid misdiagnosis ${ }^{[5]}$. In this study, D-dimer content of patients with acute myocardial infarction was obviously lower than that of patients with acute aortic dissection, which is consistent with the above research result.

Troponin is also has important clinical application value. Existing researches indicate that, troponin plays a great role in auxiliary diagnosis of acute myocardial infarction, prognostic evaluation of unstable angina pectoris and monitoring of thrombolytic therapy of acute myocardial infarction ${ }^{[6]}$. In addition, troponin can also be used as an important marker of myocardial cell damage ${ }^{[7]}$. The literatures and reports indicate that, troponin concentration can rise rapidly after the onset of acute myocardium infarction. After about 3-6h, its content level can exceed the normal value, and reach the peak after $14-20 \mathrm{~h}^{[7]}$. In combination of existing literatures and reports, troponin content of patients with acute aortic dissection is basically normal, and generally no abnormality will occur ${ }^{[8-9]}$. In this study, troponin content of patients with acute myocardial infarction was obviously higher than that of patients with acute aortic dissection, which is consistent with the above researches. In view of error possibility of applying single D-dimer or troponin to fast diagnose acute myocardial infarction and acute aortic dissection, POCT was applied in this study, and D-dimer and troponin of patients were examined. The results verified that, D-dimer and troponin content of patients with acute myocardial infarction or acute aortic dissection differed significantly, which is highly consistent with the result of Gao Chunli et al. ${ }^{[10]}$

In conclusion, D-dimer and troponin POCT can effectively identify and diagnose fatal chest pain caused by acute myocardial infarction and acute aortic dissection, provide basis for early rescue and treatment and improve patients' prognosis, so it deserves to be promoted and applied.

\section{References}

[1] Fang Z, Zhu XH, Wei X, Jiang DS.The diagnostic value of combined D-dimer with other indicators in suspected acute aortic dissection patients.Int J Cardiol. 2018 Oct 1;268:215.

[2] Sakamoto K, Yamamoto Y, Okamatsu H, Okabe M.D-dimer is helpful for differentiating acute aortic dissection and acute pulmonary embolism fromacute myocardial infarction.Hellenic J Cardiol. 2011 Mar-Apr;52(2):123-7.

[3] Nazerian P1, Morello F2, Vanni S1, Bono A3, Castelli M1, Forno D3, Gigli C1, Soardo F3, Carbone F3, Lupia E3, Grifoni S1.Combined use of aortic dissection detection risk score and D-dimer in the diagnostic workup of suspected acute aortic dissection. Int J Cardiol. 2014 Jul 15;175(1):78-82.

[4] Albini P1, Barshes NR2, Russell L1, Wu D1, Coselli JS1, Shen YH1, Allison PM3, LeMaire SA4.D-dimer levels remain elevated in acute aortic dissection after 24 h.J Surg Res. 2014 Sep;191(1):58-63.

[5] Hazui H, Fukumoto H, Negoro N, Hoshiga M, Muraoka H, Nishimoto M, Morita H, Hanafusa T..Simple and useful tests for discriminating between acute aortic dissection of the ascending aorta and acute myocardial infarction in the emergency setting..Circ J. 2005 Jun;69(6):677-82..

[6] Sakamoto K, Yamamoto Y, Okamatsu H, Okabe M.D-dimer is helpful for differentiating acute aortic dissection and acute pulmonary embolismfrom acute myocardial infarction.Hellenic J Cardiol. 2011 Mar-Apr;52(2):123-7.

[7] Catlow J1, Cross T1.A case of painless acute Type-A thoracic aortic dissection.Age Ageing. 2015 Jan;44(1):171-2.

[8] Weber T, Rammer M, Auer J, Maurer E, Aspöck G, Eber B.Plasma concentrations of D-dimer predict mortality in acute type A aortic dissection.Heart. 2006 Jun;92(6):836-7.

[9] Wen D1, Du X, Dong JZ, Zhou XL, Ma CS.Value of D-dimer and C reactive protein in predicting inhospital death in acute aortic dissection.Heart. 2013 Aug;99(16):1192-7.

[10] Tian L1, Fan X, Zhu J, Liang Y, Li J, Yang Y.Plasma D-dimer and in-hospital mortality in patients with Stanford type A acute aortic dissection.Blood Coagul Fibrinolysis. 2014 Mar;25(2):161-6. 